\title{
Identification of Hydrolysable Tannins in the Reaction Zone of Eucalyptus nitens Wood by High Performance Liquid Chromatography- Electrospray Ionisation Mass Spectrometry
}

\author{
K. M. Barry, ${ }^{1,2} *$ N. W. Davies ${ }^{3}$ and C. L. Mohammed ${ }^{1,2,4}$ \\ ${ }^{1}$ CRC for Sustainable Production Forestry, GPO Box 252-12, Hobart, Tasmania 7001, Australia \\ ${ }^{2}$ School of Agricultural Science, University of Tasmania, GPO Box 252-54, Hobart, Tasmania 7001, Australia \\ ${ }^{3}$ Central Science Laboratory, University of Tasmania, GPO Box 252-74, Hobart, Tasmania 7001, Australia \\ ${ }^{4}$ CSIRO Forestry and Forest Products, GPO Box 252-12, Hobart, Tasmania, 7001 Australia
}

\begin{abstract}
The first detailed analysis of the phenolic constituents of the reaction zones (tissue of antimicrobial defence) from the sapwood of a Eucalyptus spp. is presented. Plantation-grown Eucalyptus nitens trees with stem decay resulting from pruning wounds were sampled and extracts were prepared from healthy sapwood and from reaction zone tissue. Analysis by HPLC with ESI-MS revealed that a diverse range of hydrolysable tannins are present in both healthy sapwood and in reaction zone extracts, including over 30 gallotannins, ellagitannins and phenols. Eight tannins were unequivocally identified, including the gallotannins tri- $O$ galloyl- $\beta$-D-glucose, tetra- $O$-galloyl- $\beta$-D-glucose and penta- $O$-galloyl- $\beta$-D-glucose, and the ellagitannins pedunculagin, tellimagrandin I, casuarinin, casuarictin and tellimagrandin II. The phenols gallic acid, ellagic acid and catechin were also identified. The ellagitannins (particularly pedunculagin) are considerably more abundant in the reaction zone than in the healthy sapwood and may contribute to the effectiveness of the reaction zone as an antimicrobial barrier. Copyright (C) 2001 John Wiley \& Sons, Ltd. Keywords: HPLC; electrospray MS; tannin; ellagitannin; gallotannin; antimicrobial; Eucalyptus sp.
\end{abstract}

\section{INTRODUCTION}

The term hydrolysable tannin refers to both ellagitannins and gallotannins. Ellagitannins are esters of hexahydroxydiphenoyl (HHDP) groups with a sugar core (usually glucose) and often contain galloyl groups. Gallotannins consist of a sugar substituted only with galloyl groups. For reviews of hydrolysable tannins see Haslam (1989) and Okuda et al. (1995).

A role in antimicrobial defence has often been implicated for hydrolysable tannins; for example, durable eucalypt and oak heartwoods are rich in ellagitannins (Scalbert, 1992). Hillis (1987) states that gallotannins are found in the heartwood of Castanea and some Quercus species, while large amounts of ellagitannins exist in the heartwoods of some Eucalyptus and Quercus species. Approximately 20 ellagitannins have been reported from the heartwood of Eucalyptus species (e.g. Seikel and Hillis, 1970; Hillis and Yazaki, 1973; Hart and Hillis, 1974; Yazaki et al., 1993). Other studies have noted the presence of numerous ellagitannins in eucalypt branch wood but have not identified individual compounds (Conde et al., 1995; Cadahia et al., 1997). Some heartwood ellagitannins show anti-fungal activities in

* Correspondence to: K. M. Barry, CRC for Sustainable Production Forestry, GPO Box 252-12, Hobart, Tasmania 7001, Australia.

E-mail: karen.barry@ffp.csiro.au

Contract/grant sponsor: Tasmanian Forest Research Council. bioassays, including ellagitannin D-6 and D-13 from Eucalyptus species (Hart and Hillis, 1974). Tannin toxicity to micro-organisms has been explained by reference to the characteristic ability of tannins to bind proteins (Field and Lettinga, 1992; Kawamoto et al., 1997) or metals (Mila et al., 1996) and their antioxidant nature (Okamura et al., 1993; Hagerman et al., 1998).

Reaction zones are a major component of active antimicrobial defence in the secondary xylem of woody plants (Shain, 1979; Pearce 1996). The reaction zone forms at the interface between living sapwood and fungal infection, and is able to restrict or slow fungal decay. This function has been attributed to the deposition of phenolics and polyphenolics, as well as to microenvironmental factors (Boddy and Rayner, 1983; Yamada et al., 1988; Pearce, 1996). The reaction zone in Eucalyptus nitens is purple-blue in colour, is rich in polyphenols and appears to be resistant to colonisation by decay fungi (Barry et al., 2000). The composition of phenolics in the reaction zone has never been studied in any Eucalyptus spp.

The study of hydrolysable tannins has developed substantially in the past two decades (Haslam, 1989; Okuda et al., 1995). Molecular weight information has been obtained by fast-atom bombardment MS of purified compounds (Okuda et al., 1995). The majority of tannins are beyond the range of GC analysis, and HPLC has been necessary for the determination of complex tannin extracts. Combining HPLC with MS has recently provided a powerful tool in the analysis of polyphenolics 

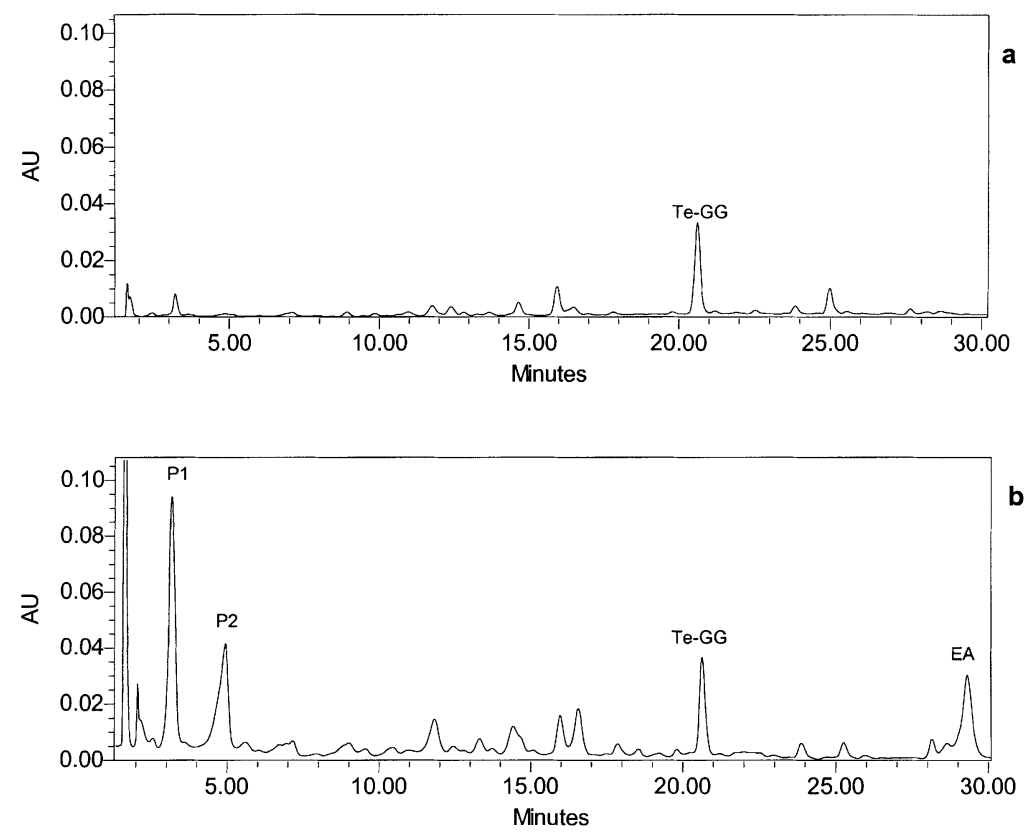

Figure 1. HPLC chromatograms of extracts $(70 \%$ acetone) of wood of Eucalyptus nitens detected at $280 \mathrm{~nm}$ showing (a) healthy sapwood, and (b) reaction zone. Key to peak identities: P1 and P2, anomers of pedunculagin; Te-GG, tetra-galloylglucose; EA, ellagic acid: acetone coelutes with P1. (For chromatographic protocols see Experimental section.)

from crude and purified extracts by detecting negative ions produced by electrospray ionisation (ESI) (Nawwar et al., 1997; Puech et al., 1999). However, little MS-MS detail of hydrolysable tannins has yet been provided.

The present study describes the application of HPLCESI-MS to elucidate the major components of the complex extract from the reaction zone of E. nitens. By reference to authentic standards of phenols, gallotannins and ellagitannins, 11 compounds have been unequivocally identified in crude extracts.

\section{EXPERIMENTAL}

Sample preparation. A number of plantation trees of Eucalyptus nitens between 5 and 7 years old were felled at various sites in Tasmania and those with decay columns interfaced with reaction zones were sampled. Extracts were prepared from fresh wood by obtaining chisel shavings (100-500 mg) of the reaction zone and of healthy sapwood tissue. Samples were extracted with $70 \%$ aqueous acetone for $24 \mathrm{~h}$ in the dark at $4^{\circ} \mathrm{C}$. The extract was then transferred to a microcentrifuge tube, centrifuged to remove solids and then appropriately diluted for LC-MS. Samples were stored at $-20^{\circ} \mathrm{C}$ and analysed as soon as possible (within a week). Injections of $2 \mu \mathrm{L}$ sample were made, with pairs of extracts at equivalent concentrations (based on the weight of wood tissue) where semi-quantitative measurements were to be made. Typical yields of extract (as a percentage of fresh weight) were approximately $6 \%$ for reaction zone extracts and $1.5 \%$ for healthy sapwood extracts.

Authentic standards. The tannin standards were pedunculagin, pentagalloylglucose, corilagin, ellagitannins D-6 and D-13, 1,2,6-tri- $O$-galloyl- $\beta$-D-glucose, 1,2,3,6-tetra$O$-galloyl- $\beta$-D-glucose, tellimagrandin I and II, casuarictin, casuarinin, and rugosin A and B [generous gifts from T. Yoshida (CSIRO, Melbourne, Australia) and from Y. Yazaki (Okayama University, Japan)]. The phenol standards were gallic acid, ellagic acid and catechin (Sigma, St Louis, MO, USA). Standards were accurately weighed and dissolved in $95 \%$ aqueous acetone at concentrations of $0.10 \mathrm{mg} / 100 \mu \mathrm{L}$. For later use, standards were dried under a stream of nitrogen at room temperature, stored at $-20^{\circ} \mathrm{C}$ and reconstituted when further required.

HPLC-ESI-MS. HPLC separations were carried out on a Waters Alliance 2690 chromatograph employing a Waters Nova Pak $\mathrm{C}_{18}$ column $(150 \times 3.9 \mathrm{~mm}$ i.d. $)$. The mobile phase consisted of solvent A (water:acetic acid, 98:2) and solvent B (methanol:acetic acid, 98:2); initial conditions were $95 \% \mathrm{~A}$ and $5 \% \mathrm{~B}$ followed by a linear gradient to $46 \% \mathrm{~A}$ and $54 \% \mathrm{~B}$ over $40 \mathrm{~min}$ at a flow-rate of $0.8 \mathrm{~mL} / \mathrm{min}$, before returning to initial conditions with $12 \mathrm{~min}$ re-equilibration between samples. Compounds were detected using a Waters model 996 photodiode array detector monitoring over the range $240-400 \mathrm{~nm}$ at a resolution of $1.2 \mathrm{~nm}$.

MS was carried out on a Finnigan LCQ (San Jose, CA, USA) with an electrospray ion source, using LCQ Navigator version 1.2 software. The instrument was operated in the negative ion mode, scanning from $\mathrm{m} / \mathrm{z} 125$ to 1500 , with an AGC target value of $2 \times 10^{7}$ and maximum ion injection time of $100 \mathrm{~ms}$. Operating conditions were: sheath gas, $90 \mathrm{psi}$; auxiliary gas, 50 psi; ESI needle voltage, $4.5 \mathrm{kV}$; capillary temperature, $270^{\circ} \mathrm{C}$; capillary voltage, $-30 \mathrm{~V}$. Data-dependent MSMS spectra were routinely acquired from the most intense ion in the spectrum with a default collision energy of $30 \%$ and a peak isolation width of $3 \mathrm{amu}$. 

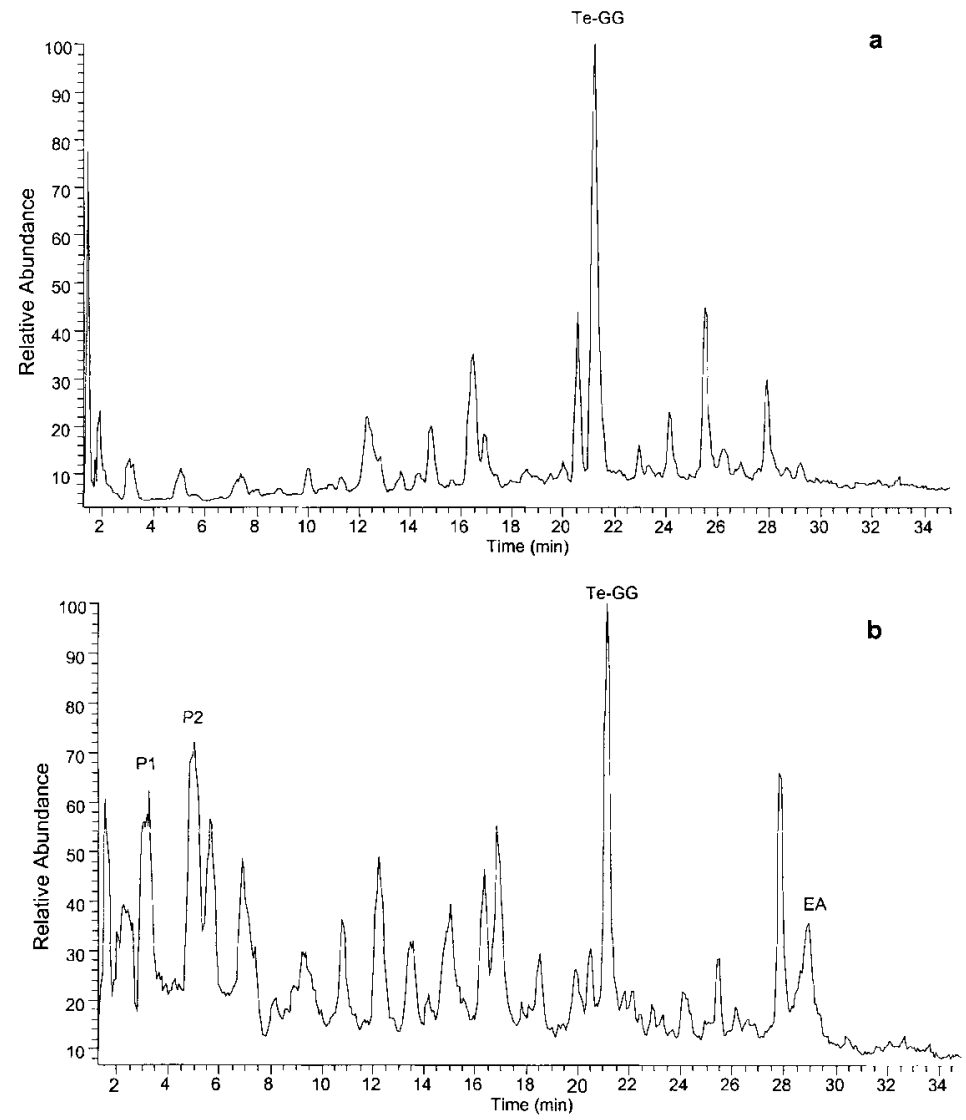

Figure 2. Total ion current chromatograms of extracts $(70 \%$ acetone) of wood of Eucalyptus nitens showing (a) healthy sapwood, and (b) reaction zone. (Refer to the legend of Fig. 1 for key to peak identities and chromatographic protocols).
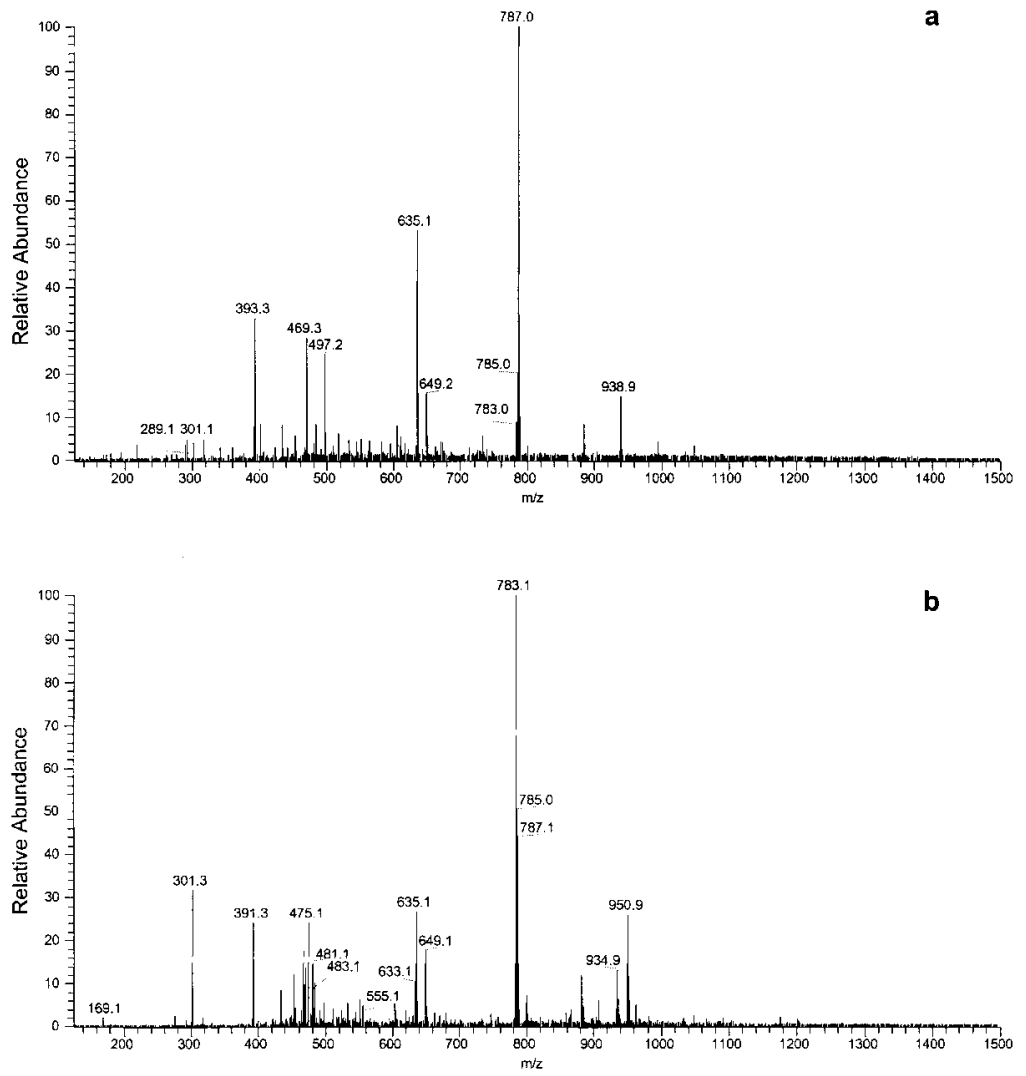

Figure 3. The summed mass spectra in the range of $\mathrm{m} / \mathrm{z} 125-1500$ for extracts ( $70 \%$ acetone) of wood of Eucalyptus nitens showing (a) healthy sapwood, and (b) reaction zone. 


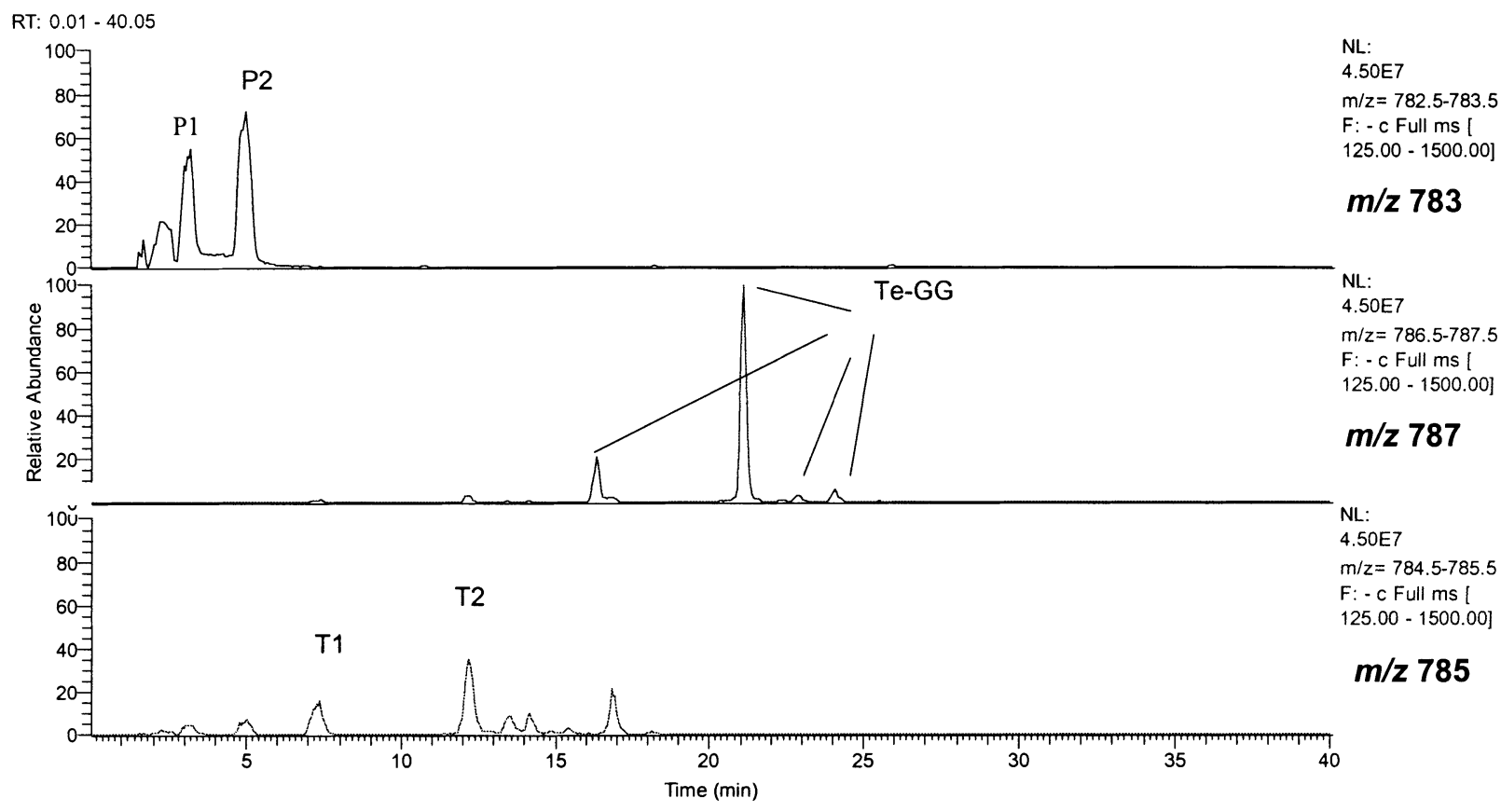

Figure 4. Mass chromatograms of three selected $[\mathrm{M}-\mathrm{H}]^{-}$ions in the extract of the reaction zone of Eucalyptus nitens. Key to peak identities: P1 and P2, anomers of pedunculagin; Te-GG, tetra- $O$-galloyl- $\beta$-D-glucose isomers; T1 and T2, tellimagrandin I anomers. (For chromatographic protocols see Experimental section.)

\section{RESULTS AND DISCUSSION}

\section{Methodology and interpretation}

The polar nature and complexity of the extracts from the reaction zone of $E$. nitens limited successful HPLC resolution. The difficulty in obtaining good HPLC separation of wood tannins is evidenced from previous studies (e.g. Scalbert et al., 1988; Yazaki et al., 1993). However, long and time-consuming HPLC programs (e.g in excess of $80 \mathrm{~min}$ ) can be employed to improve separation (Cadahia et al., 1997). Trials of a range of HPLC programs did not substantially improve the separation of the extracts from $E$. nitens, precluding peak identification based on retention times and UV spectra alone.

Figure 1(a) and (b) shows that the HPLC chromatogram (detected at $280 \mathrm{~nm}$ ) of the extract of the reaction zone is more complex than that of the healthy sapwood.
Figure 2(a) and (b) presents the corresponding total ion chromatograms from the HPLC-MS analyses of these extracts, and indicates that the mass range employed (up to $\mathrm{m} / \mathrm{z} 1500$ ) is appropriate for the components detected by UV as similar peaks are observed in both spectra. MS revealed that many compounds in the extract co-elute and that peaks cannot be interpreted by UV detection alone using current HPLC systems.

In order to gain an overview of the molecular weight range of the major compounds present in each extract, a summed MS was determined between $\mathrm{m} / \mathrm{z} 125$ and 1500 [Fig. 3(a) and (b)]. Of the major ions present, those representing molecular weights corresponding to the tannin and phenolic standards available were selected and the retention times and negative ion ESI-MS characteristics were determined. Compounds were unequivocally identified based on matching mass chromatograms generated for specific $[\mathrm{M}-\mathrm{H}]^{-}$ions (e.g. Fig. 4), on MS of the $[\mathrm{M}-\mathrm{H}]^{-}$ions at specific retention times (e.g. Fig. 5)

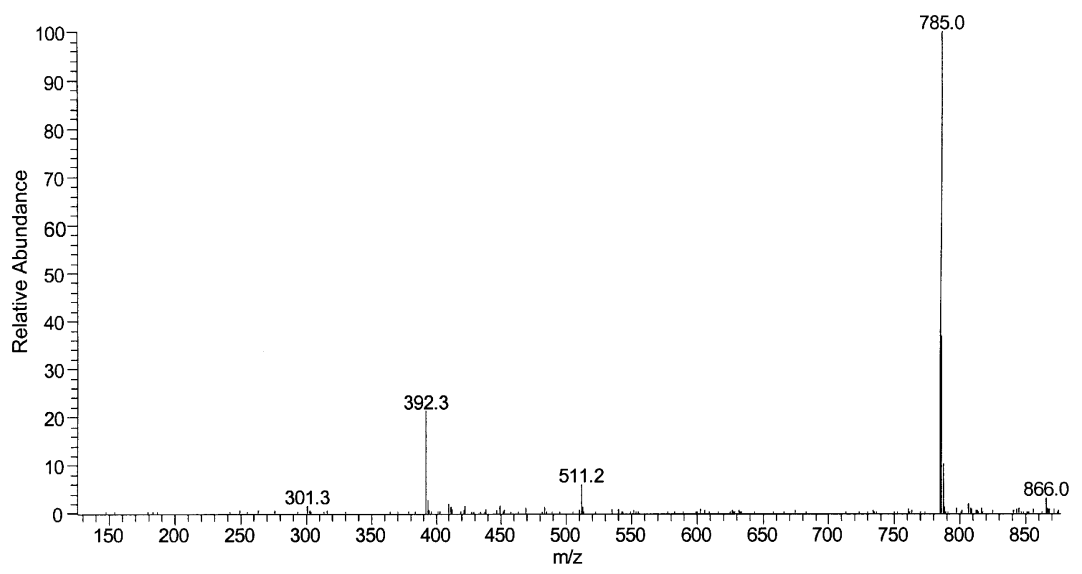

Figure 5. Mass spectrum of tellimagrandin I $\left([\mathrm{M}-\mathrm{H}]^{-}=785 ;[\mathrm{M}-2 \mathrm{H}]^{2-}=392\right)$ from the extract of the reaction zone of Eucalyptus nitens. 

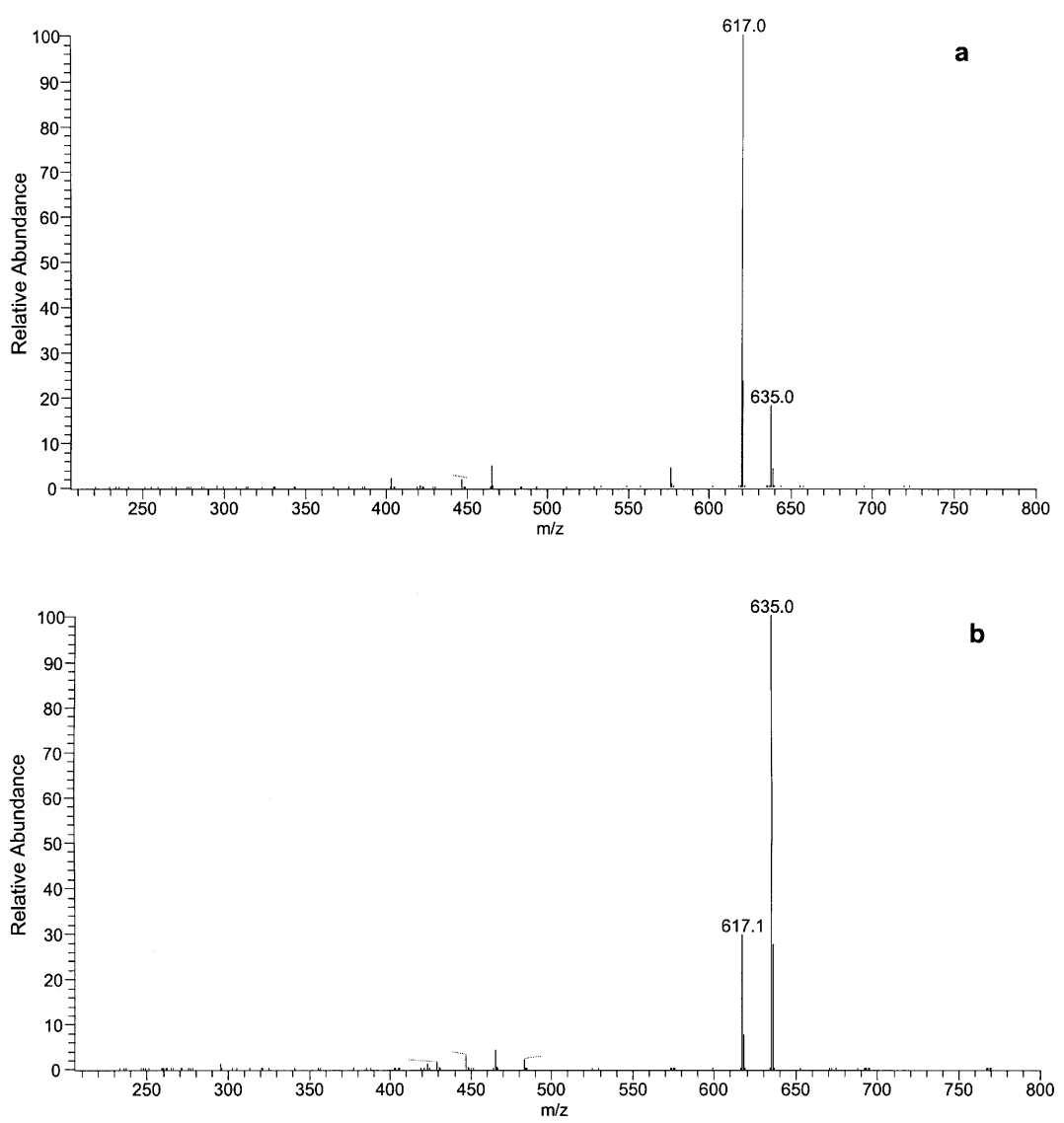

Figure 6. MS-MS daughters of the $[\mathrm{M}-\mathrm{H}]$-ion at $m / z$ 787, (a) from the 1,2,3,6tetra-galloyl- $\beta$-D-glucose standard at $21.08 \mathrm{~min}$, and (b) from the reaction zone extract peak at $21.08 \mathrm{~min}$.

and, subsequently, on MS-MS of daughter ions from a selected parent ion [e.g. Fig. 6(a)]. The assignment of numerous other peaks as positional isomers (e.g. various di-, tri- and tetra-galloylglucoses) was also possible. Furthermore, the degree of substitution of HHDP and galloyl groups in unknown compounds could be assigned. Interpretation of MS-MS data from first principles indicated the presence of galloyl groups (losses of 152 and 170 from the $[\mathrm{M}-\mathrm{H}]^{-}$ion), and/or HHDP groups (loss of 302 from the $[\mathrm{M}-\mathrm{H}]^{-}$ion and the presence of an ion at $\mathrm{m} / \mathrm{z} 301)$. Loss of 44 from the $[\mathrm{M}-\mathrm{H}]^{-}$ion was characteristic of a free carboxyl (e.g. on a trisgalloyl group) and abundant losses of 18 (water) from the $[\mathrm{M}-\mathrm{H}]^{-}$ion was characteristic of $\mathrm{C}$-glucosidic ellagitannins.

The characteristic trait that a free anomeric hydroxyl group at $\mathrm{C} 1$ of the glucose core of an ellagitannin molecule results in two chromatographic peaks was also a feature for identification (Hatano et al., 1988). Addition of sodium borohydride to aqueous extracts reduces tannins with anomeric hydroxyls, resulting in a single peak (Hatano et al., 1988) which also presents a tool for identification. Another characteristic of the ESI-MS of most tannins is that doubly-charged ions (i.e. [M $2 \mathrm{H}]^{2-}$ ) are also formed (e.g. Fig. 5).

\section{Compound identification}

Table 1 outlines some 30 major and minor compounds discriminated from the E. nitens extracts, showing relative proportions (based on MS peak area) which have been classified arbitrarily as described. The sensitive MS system used in the present study also detected a multitude of smaller peaks which have not been analysed. Five ellagitannins (pedunculagin, tellimagrandin I and II, casuarinin and casuarictin), three gallotannins (trigalloylglucose, tetra-galloylglucose and penta-galloylglucose) and three phenols (catechin, gallic acid and ellagic acid) were unequivocally identified. The structure of an abundant ellagitannin (pedunculagin) is shown in Fig. 7 and a variety of gallotannins are illustrated in Fig. 8. A number of assumed positional isomers and unidentified compounds are also presented in Table 1 and are discussed below.

Treatment of reaction zone extracts of E. nitens (prepared as aqueous solutions) with sodium borohydride revealed that at least three prominent ellagitannins that form anomers are present, since three prominent "new" reduction product peaks were detected (K. M. Barry, unpublished results). Two of these anomer-forming ellagitannins are pedunculagin and tellimagrandin I, and the third may be a compound with $[\mathrm{M}-\mathrm{H}]^{-}$at $\mathrm{m} / \mathrm{z}$ 951.

The general substitution pattern of HHDP and galloyl groups for a number of compounds in Table 1 has been determined from molecular weight information and characteristic MS-MS daughter ions. Where molecular weights of hydrolysable tannins differ by two, it can be related to the difference between either an HHDP group or two galloyl groups. For example, by coupling two adjacent galloyl groups of the four from tetra-galloylglucose (788) by intramolecular oxidation, tellimagrandin I (786) would be formed. Coupling of the two remaining 
Table 1. Major and minor hydrolysable tannins present in extracts of wood of Eucalyptus nitens as determined by HPLC-ESIMS

\begin{tabular}{|c|c|c|c|c|}
\hline \multirow[b]{2}{*}[\mathrm{M}-\mathrm{H}]{$^{-}$} & \multirow[b]{2}{*}{ Retention time (min) } & \multirow[b]{2}{*}{ Compound } & \multicolumn{2}{|c|}{ Relative abundance $^{a}$} \\
\hline & & & Healthy sapwood & Reaction zone \\
\hline 169 & 2.79 & Gallic acid ${ }^{b}$ & $\operatorname{tr}$ & + \\
\hline 289 & 10.03 & Catechin ${ }^{\mathrm{b}}$ & + & $\operatorname{tr}$ \\
\hline 301 & 28.93 & Ellagic acid ${ }^{\mathrm{b}}$ & + & ++ \\
\hline 481 & 1.55 & HHDP-glucose & $\operatorname{tr}$ & +++ \\
\hline \multirow[t]{5}{*}{483} & 8.83 & Di-galloylglucose ${ }^{v}$ & $\operatorname{tr}$ & $\operatorname{tr}$ \\
\hline & 10.67 & Di-galloylglucose ${ }^{w}$ & $\operatorname{tr}$ & $\operatorname{tr}$ \\
\hline & 12.00 & Di-galloylglucose $e^{x}$ & $\operatorname{tr}$ & $\operatorname{tr}$ \\
\hline & 13.59 & Di-galloylglucose ${ }^{y}$ & $\operatorname{tr}$ & $\operatorname{tr}$ \\
\hline & 14.49 & Di-galloylglucose ${ }^{z}$ & $\operatorname{tr}$ & $\operatorname{tr}$ \\
\hline 633 & 9.61 & HHDP-GG & $\operatorname{tr}$ & ++ \\
\hline \multirow[t]{5}{*}{635} & 12.46 & Tri-galloylglucosew b & + & + \\
\hline & 14.75 & Tri-galloylglucose $\mathrm{e}^{\mathrm{b}}$ & ++ & ++ \\
\hline & 16.23 & Tri-galloylglucose $\mathrm{y}^{\mathrm{b}}$ & ++ & ++ \\
\hline & 16.39 & $1,2,6$ - Tri-galloylglucose $\mathrm{b}^{\mathrm{b}}$ & $\operatorname{tr}$ & $\operatorname{tr}$ \\
\hline & 16.79 & Tri-galloylglucose $\mathrm{e}^{\mathrm{b}}$ & + & + \\
\hline 649 & 27.82 & Methyl-(tri-galloylglucose) & ++ & +++ \\
\hline \multirow{2}{*}{783} & 2.40 & Di-HHDP-glucose & $\operatorname{tr}$ & ++ \\
\hline & $3.16 / 5.00$ & Pedunculagin ${ }^{b}$ & + & ++++ \\
\hline \multirow[t]{4}{*}{785} & $7.27 / 12.19$ & Tellimagrandin $\mathrm{I}^{\mathrm{b}}$ & ++ & +++ \\
\hline & 13.52 & HHDP-di-galloylglucose ${ }^{x}$ & $\operatorname{tr}$ & ++ \\
\hline & 14.19 & HHDP-di-galloylglucose ${ }^{y}$ & + & ++ \\
\hline & 16.85 & HHDP-di-galloylglucose ${ }^{z}$ & + & ++ \\
\hline \multirow[t]{4}{*}{787} & 16.29 & Tetra-galloylglucose $\mathrm{w} \mathrm{b}^{\mathrm{b}}$ & + & + \\
\hline & 21.08 & Tetra-galloylglucose $\mathrm{x}^{\mathrm{b}}+1,2,3,6$-tetra-galloylglucose & ++ & +++ \\
\hline & 22.86 & Tetra-galloylglucose $\mathrm{y}^{\mathrm{b}}$ & $\operatorname{tr}$ & $\operatorname{tr}$ \\
\hline & 24.04 & Tetra-galloylglucose $\mathrm{z}^{\mathrm{b}}$ & $\operatorname{tr}$ & $\operatorname{tr}$ \\
\hline \multirow[t]{3}{*}{935} & 9.32 & Casuarinin & 0 & + \\
\hline & 15.02 & Casuarictin & 0 & + \\
\hline & 23.10 & Di-HHDP-galloylglucose & $\operatorname{tr}$ & + \\
\hline \multirow[t]{4}{*}{937} & 16.91 & HHDP-tri-galloylglucose ${ }^{x}$ & $\operatorname{tr}$ & + \\
\hline & 18.02 & Tellimagrandin $\mathrm{II}^{\mathrm{b}}$ & $\operatorname{tr}$ & + \\
\hline & 19.24 & HHDP-tri-galloylglucose ${ }^{y}$ & $\operatorname{tr}$ & + \\
\hline & 32.95 & HHDP-tri-galloylglucose ${ }^{z}$ & + & 0 \\
\hline 939 & 25.42 & Penta-galloylglucose ${ }^{b}$ & $\operatorname{tr}$ & + \\
\hline \multirow[t]{2}{*}{951} & $5.64 / 10.84$ & (Trisgalloyl)-HHDP-glucose ${ }^{y}$ & $\operatorname{tr}$ & +++ \\
\hline & 6.88 & (Trisgalloyl)-HHDP-glucose ${ }^{z}$ & 0 & +++ \\
\hline
\end{tabular}

galloyl groups from tellimagrandin I would produce pedunculagin (784) (Fig. 7). The loss of two hydrogen atoms resulting from bonding of galloyl groups may occur similarly for other structures. The strong $[\mathrm{M}-\mathrm{H}]^{-}$

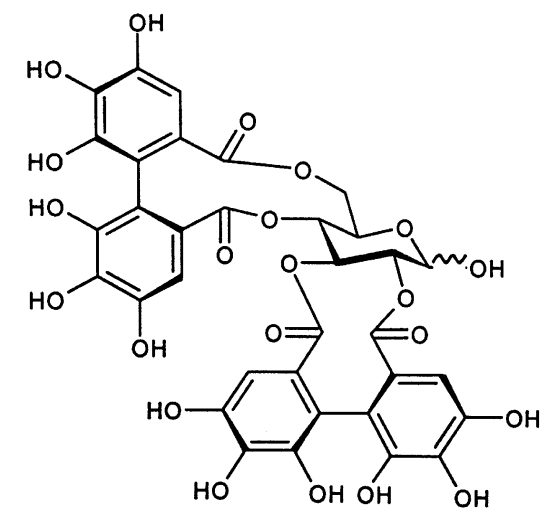

Figure 7. The structure of pedunculagin $(2,3 ; 4,6-$ di-HHDPglucose). at $m / z 481$ in the reaction zone samples has been assigned as an HHDP-glucose, based on molecular weight and the presence of an intense daughter ion at $\mathrm{m} / \mathrm{z}$ 301. The compound with $[\mathrm{M}-\mathrm{H}]^{-}$ion at $m / z 633$, abundant in the reaction zone samples, has been assigned as an HHDP-

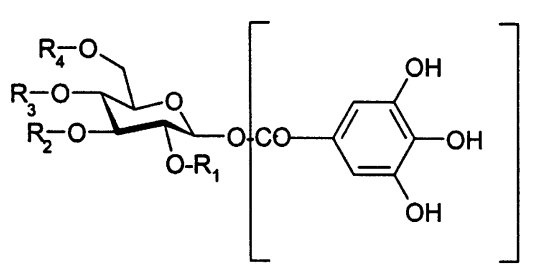

Figure 8. Examples of gallotannins with various numbers of galloyl groups (G; substituent shown in brackets) linked to the glucose core. $\beta$-Glucogallin (1), $\mathrm{R}_{1}=\mathrm{R}_{2}=\mathrm{R}_{3}=\mathrm{R}_{4}=\mathrm{H} ; 1$,6Di-O-galloyl- $\beta$-D-glucose (4), $\mathrm{R}_{1}=\mathrm{R}_{2}=\mathrm{R}_{3}=\mathrm{H}, \mathrm{R}_{4}=\mathrm{G}$; 1,2,6-TriO-galloyl- $\beta$-D-glucose (6), $\mathrm{R}_{1}=\mathrm{R}_{4}=\mathrm{G}, \mathrm{R}_{2}=\mathrm{R}_{3}=\mathrm{H} ; 1,2,3,6$ Tetra-O-galloyl- $\beta$-D-glucose (4), $R_{1}=R_{2}=R_{4}=G, R_{3}=H ; 1,2,3$, 4,6-Penta-O-galloyl- $\beta$-D-glucose (1), $\mathrm{R}_{1}=\mathrm{R}_{2}=\mathrm{R}_{3}=\mathrm{R}_{4}=\mathrm{G}$. The number of $\beta$ positional isomers expected for each gallotannin is in brackets. 
galloylglucose (Table 1), from the MS-MS evidence of intense ions at $\mathrm{m} / \mathrm{z} 301$ and 463. The minor $[\mathrm{M}-\mathrm{H}]^{-}$ ions at $m / z, 483$ have been assigned as di-galloylglucoses.

Casuarinin and casuarictin (both with an $[\mathrm{M}-\mathrm{H}]^{-}$ion at $\mathrm{m} / \mathrm{z}$ 935) could be distinguished by their retention times and also by their MS-MS spectra. The MS-MS daughters of the casuarinin $[\mathrm{M}-\mathrm{H}]^{-}$ion showed a strong ion $(30 \%$ relative abundance) resulting from a loss of water, owing to its open glucose ring structure ( $C$-glucoside rather than $O$-glucoside). For casuarictin this ion was only $0.2 \%$ relative to the strongest ion corresponding to the loss of 302 as discussed above. The peak eluting at $2.40 \mathrm{~min}$ prior to the two pedunculagin anomers at $m / z 783$ (Fig. 4) also showed a strong ion (20\% relative abundance) resulting from a loss of water from the $[\mathrm{M}-\mathrm{H}]^{-}$ion and therefore may be the $\mathrm{C}$-glucoside version of pedunculagin.

Based on molecular weight and MS-MS data, the three abundant peaks with $[\mathrm{M}-\mathrm{H}]^{-}$at $m / z 951$ in the reaction zone samples (Table 1) would appear to be tannins with one HHDP group and one trisgalloyl group (e.g. valoneoyl, tergalloyl, macaronyl). All three peaks lose 44 from the $[\mathrm{M}-\mathrm{H}]^{-}$ion, consistent with a free carboxyl group, as found in a compound such as praecoxin A. As mentioned, the two peaks at $m / z, 951$ eluting at 5.64 and $10.84 \mathrm{~min}$ (Table 1) may be anomeric forms of one compound, therefore suggesting a free hydroxyl at the glucose $\mathrm{C} 1$. The single peak at 6.88 min may be a variation on this which involves bonding at the $\mathrm{C} 1$ position.

The main peak of tetra-galloylglucose at $21.08 \mathrm{~min}$ in the E. nitens extracts (see Fig. 4) is likely to represent two isomers. The 1,2,3,6-tetra- $O$-galloyl- $\beta$-D-glucose standard eluted at this time and its MS-MS spectrum [Fig. 6(a)] showed two major daughter ions of which that at $\mathrm{m} / \mathrm{z} 617$ was 5-fold more abundant than the ion at $\mathrm{m} / \mathrm{z} 635$. However, the MS-MS spectrum of the E. nitens peak at 21.08 min [Fig. 6(b)] revealed that the daughter ions were in reverse proportion and, therefore, came largely from a different isomer. This latter ratio was consistent in all reaction zone and healthy sapwood extracts analysed. Four major tetra-galloylglucose isomers were detected in the E. nitens extracts (Fig. 4 and Table 1). As the possible number of $\beta$ isomers for tetra-galloylglucose is four (see Fig. 8), it would be surprising if the 1,2,3,6-isomer were not present, and therefore it may be making a small contribution to the main peak in the E. nitens extracts. The number of possible positional isomers of the other gallotannins (Fig. 8) correlates similarly, but not exactly, to the number of peaks representing each gallotannin isomer in the wood extracts (Table 1).

\section{Tannin levels and biological significance}

For simplicity, compound abundance has been classified into groups based on MS peak areas (from a typical set of samples) as detailed in Table 1. The abundance of pedunculagin was found to be increased approximately 50 -fold in the reaction zone compared to the healthy sapwood. Many other ellagitannins were also present at greatly increased levels in the reaction zone. However, there was at most only a slight increase in the levels of the gallotannins in the reaction zone samples. This suggests that the ellagitannins are more important in the antimicrobial effectiveness of the reaction zone. Gallotannins such as penta- $O$-galloyl- $\beta$-D-glucose may be important as ellagitannin precursors (Hatano et al., 1986; Haslam,
1989). Interestingly, preliminary HPLC-MS studies of $E$. nitens leaves have shown that a range of gallotannins is present in light stressed leaves compared to normal leaves, but few/no ellagitannins (D. C. Close and N. W. Davies, unpublished results).

Haslam (1989) has classified plants with penta- $O$ galloyl- $\beta$-D-glucose and one or more of tellimagrandin II, tellimagrandin I, casuarictin, potentillin and pedunculagin as having "group 2B" phenolic metabolites. Metabolites from the wood of $E$. nitens therefore fall within this grouping, as has been found for the Myrtaceae family in general (Haslam, 1989). The range of closely related hydrolysable tannins and isomers found in E. nitens wood is a common occurrence and may prove advantageous for defence (Zucker, 1983). For example, tannin-protein binding may be an important mechanism to halt fungal metabolism, and a range of tannin structures may promote a variety of specific tannin-protein interactions.

As casuarictin and casuarinin (and a compound at $\mathrm{m} / \mathrm{z}$ 951 eluting at $6.88 \mathrm{~min}$ ) were not detected in the $E$. nitens healthy sapwood, these compounds may be produced $d e$ novo in the reaction zone from pedunculagin (Okuda $e t$ al., 1995). All other tannins are detectable in at least trace amounts in the healthy sapwood (Table 1). Increases in the concentration of tannins of the magnitude found in the $E$. nitens reaction zone would be expected to confer biological activity: for example, Hart and Hillis (1972) found that even a 2-fold increase of white oak heartwood extracts resulted in antifungal inhibition to Poria monticola in bioassay.

Catechin has not been previously detected in Eucalyptus sapwood (Y. Yazaki, personal communication), and this may be because of a higher detection sensitivity in this study, or it may represent a novel finding for $E$. nitens. The decreased levels of catechin in the reaction zone may be due to the formation of condensed tannins, and the purple/blue colour of the zone is suggestive of condensed tannins. However these compounds were not analysed in this study and there was no evidence of condensed tannins such as epicatechin gallate.

In conclusion, HPLC-MS provides a powerful and relatively quick technique with which to elucidate the complex nature of crude wood extracts. It provides a solid basis for identification with reference to retention times, unequivocal molecular weight assignment and characteristic daughter ions. At least 30 hydrolysable tannins (including structural isomers) were detected in the wood extracts of E. nitens. Eight of the hydrolysable tannins and three phenols were unequivocally identified by comparison with standards. The ellagitannins were orders of magnitude more abundant in the reaction zone compared to healthy sapwood. The ellagitannin pedunculagin is particularly concentrated in the reaction zone and may play an important role in its antimicrobial function.

\section{Acknowledgements}

One of us (K.M.B.) acknowledges the assistance of an Australian Postgraduate Award with industry funding from the Tasmanian Forest Research Council. The authors are extremely grateful to Y. Yazaki (CSIRO, Melbourne, Australia) for standards of pedunculagin, pentagalloylglucose, corilagin, and ellagitannins D-6 and D-13, and to T. Yoshida (Okayama University, Japan) for standards of tri-galloyl glucose, tetra-galloylglucose, tellimagrandin I and II, casuarictin, casuarinin, and rugosin $\mathrm{A}$ and $\mathrm{B}$. 


\section{REFERENCES}

Barry KM, Pearce RB, Mohammed CL. 2000. Properties of reaction zones associated with decay from pruning wounds in plantation-grown Eucalyptus nitens. Eur J Pathol 30: 233-245.

Boddy L, Rayner ADM. 1983. Origins of decay in living deciduous trees: the role of moisture content and a reappraisal of the expanded concept of tree decay. New Phytol 94: 623-641.

Cadahia E, Conde E, Garcia-Vallejo MC, Fernandez de Simon B. 1997. Tannin composition of Eucalyptus camaldulensis, E. globulus and E. rudis I. Wood. Holzforschung 51: 119-124.

Conde E, Cadahia E, Garcia-Vallejo MC, Tomas-Barberan F. 1995. Low molecular weight polyphenols in wood and bark of Eucalyptus globulus. Wood Fiber Sci 27: 379-383.

Field JA, Lettinga G. 1992. Toxicity of tannic compounds to microorganisms. In Plant Polyphenols, Hemingway RW, Laks PE (eds). Plenum Press: New York; 673-692.

Hagerman AE, Riedl KM, Jones A, Sovik KN, Ritchard NT, Hartzfeld PW, Riechel TL. 1998. High molecular weight plant polyphenolics (tannins) as biological antioxidants. $J$ Agric Food Chem 46: 1887-1892.

Hart JH, Hillis WE. 1972. Inhibition of wood-rotting fungi by ellagitannins in the heartwood of Quercus alba. Phytopathology 62: 620-626.

Hart JH, Hillis WE. 1974. Inhibition of wood-rotting fungi by stilbenes and other polyphenols in Eucalyptus sideroxylon. Phytopathology 64: 939-948.

Haslam E. 1989. Plant Polyphenols, Vegetable Tannins Revisited. Cambridge University Press: Cambridge.

Hatano T, Kira R, Yoshizaki M and Okuda T. 1986. Seasonal changes in the tannins of Liquidambar formasana reflecting their biogenesis. Phytochemistry 25: 27872789.

Hatano T, Yoshida T and Okuda T. 1988. Chromatography of tannins III. Multiple peaks in high-performance liquid chromatography of some hydrolyzable tannins. J Chromatogr 435: 285-295.

Hillis WE. 1987. Heartwood and Tree Exudates. Springer: Berlin.

Hillis WE, Yazaki Y. 1973. Wood polyphenols of Eucalyptus polyanthemos. Phytochemistry 12: 2969-2977.

Kawamoto H, Mizutani K, Nakatsubo F. 1997. Binding nature and denaturation of protein during interaction with galloylglucose. Phytochemistry 46: 473-478.
Mila I, Scalbert A, Expert D. 1996. Iron withholding by plant pathogens and resistance to pathogens and rots. Phytochemistry 42: 1551-1555.

Nawwar MAM, Marzouk MS, Nigge W, Linscheid M. 1997. High performance liquid chromatographic / electrospray ionisation mass spectrometric screening for polyphenolic compounds of Epilbium hirsutum - the structure of the unique ellagitannin epilobamide-A. J Mass Spectrom 32: 645-654.

Okamura H, Mimura A, Yakou Y, Niwano M, Takahra Y. 1993. Antioxidant activity of tannins and flavonoids in Eucalyptus rostrata. Phytochemistry 33: 557-561.

Okuda T, Yoshida T, Hatano T. 1995. Hydrolysable tannins and related polyphenols. Prog Chem Org Nat Prod 66: 1117.

Pearce RB. 1996. Anti-microbial defences in the wood of living trees. New Phytol 132: 203-233.

Puech J-L, Mertz C, Michon V, Guerneve CL, Doco T, du Penhoat $\mathrm{CH}$. 1999. Evolution of castalagin and vescalagin in ethanol solutions. Identification of new derivatives. $J$ Agric Food Chem 47: 2060-2066.

Scalbert A. 1992. Tannins in woods and their contribution to microbial decay prevention. In Plant Polyphenols, Hemingway RW, Laks PE (eds). Plenum Press: New York; 935952.

Scalbert A, Monties B, Favre J. 1988. Polyphenols of Quercus robur. adult tree and in vitro grown callus and shoots. Phytochemistry 27: 3483-3488.

Seikel MK, Hillis WE. 1970. Hydrolysable tannins of Eucalyptus delegatensis wood. Phytochemistry 9: 1115-1128.

Shain L. 1979. Dynamic responses of differentiated sapwood to injury and infection. Phytopathology 69: 1143-1147.

Yamada T, Tamura H, Mineo K. 1988. The responses of sugi (Cryptomeria japonica D. Don) sapwood to fungal invasion following attack by the sugi bark borer. Physiol Mol Plant Pathol 33: 429-442.

Yazaki Y, Collins PJ, Iwashina T. 1993. Extractives from blackbutt (Eucalyptus pilularis) wood which affects gluebond quality of phenolic resins. Holzforschung 47: 412-418.

Zucker WV. 1983. Tannins: Does structure determine function? An ecological perspective. Am Naturalist 121: 335365. 